\title{
Dietary intake of persons with depressive and psychotic disorders in Singapore
}

Ying Ying Lee ${ }^{1}{ }_{P h D}$, Jue Hua Lau ${ }_{B S c}$, Vanessa $\underline{\text { Seet }}{ }_{B A}$, Clare $\underline{\text { Whitton }}{ }^{2}{ }_{M S c}$, PV $\underline{\text { Asharani }}{ }^{1}{ }_{P h D}$, Fiona Devi Siva Kumar ${ }^{1}{ }_{B A}$, Peizhi Wang ${ }^{1} M P H$, Kumarasan Roystonn ${ }^{1} M S c$, Laxman Cetty ${ }^{1} B S c$, Wen Lin Teh ${ }^{1}{ }_{B S o c s c i(H o n s)}$, Swapna Verma ${ }^{3,4}{ }_{M D}$, Yee Ming $\underline{\text { Mok }}{ }^{5}$ MMed (Psych) (Spore), Mythily $\underline{\text { Subramaniam }}{ }^{1,2}$ PhD

\begin{abstract}
Introduction: Nutritional psychiatry is an emerging field of study that investigates the role of diet and nutrition in mental health. Studies conducted in the general population have linked depressive symptoms with poor dietary patterns. The aim of this study was to characterise the dietary intake and analyse the dietary pattern using the Dietary Approach to Stop Hypertension (DASH) in a sample of psychiatric patients in a multiethnic Asian nation.

Methods: Participants were recruited from an outpatient clinic and an inpatient unit at the Institute of Mental Health in Singapore. Self-reported dietary habits of a sample of psychiatric patients (N=380) were analysed using DASH. To examine the variables associated with DASH scores, a linear regression was conducted with the full sample and sociodemographic variables.

Results: Persons with depressive disorders had a mean DASH score of $21.3( \pm 4.2)$, while persons with psychotic disorders had a mean DASH score of $21.2( \pm 4.9)$. Respondents who were older $(\mathrm{B}=1.94$, $95 \%$ confidence interval $[\mathrm{CI}] 0.91-2.96, P<0.001)$, female $(\mathrm{B}=1.09,95 \% \mathrm{CI} 0.07-2.11, P=0.04)$ and economically inactive $(\mathrm{B}=1.98,95 \%$ CI $0.006-3.96, P=0.049)$ were more likely to report a higher diet quality compared with their respective counterparts, while smokers $(B=-1.39,95 \%$ CI -2.45 to -0.34 , $P=0.009)$ tended to report a lower diet quality compared with their non-smoking counterparts.

Conclusion: Dietary patterns of persons with mental disorders were characterised. A host of sociodemographic factors, and not diagnosis of mental disorders, influenced the dietary quality of people with depressive and psychotic disorders. Clinicians treating psychiatric patients need to be aware of the nuanced reasons behind poor dietary choices and provide targeted psychoeducation to specific subgroups within the patient population.
\end{abstract}

Ann Acad Med Singap 2021;50:379-89

Keywords: Depression, diet, nutritional psychiatry, psychosis, smoking

\section{INTRODUCTION}

Unhealthy diet is a modifiable risk factor in many health conditions, ${ }^{1}$ including mental disorders. ${ }^{2}$ Nutritional psychiatry is an emerging field that examines the role of diet and nutrition in mental health. ${ }^{3}$ Since its beginnings in the 2000s, a notable change in the field was a switch in focus from individual supplements and specific food intake to a more holistic assessment of one's dietary pattern. ${ }^{3}$ The rationale for this progress was the realisation that one's diet had to be understood in the context of whole nutrition intake, rather than individual components of one's diet. What one eats in excess is as important as what one does not. ${ }^{3}$

Diet has the potential to be used as a preventive and/ or adjunctive treatment for mental disorders. ${ }^{4-6}$ In the last decade, a growing body of evidence has associated poor dietary patterns with depressive symptoms in the general population, ${ }^{7}$ women ${ }^{8}$ and college students ${ }^{9}$ across various parts of the world. Indeed, there are at least 3 meta-analyses that confirmed the findings that

\footnotetext{
${ }^{1}$ Research Division, Institute of Mental Health, Singapore

${ }^{2}$ Saw Swee Hock School of Public Health, National University of Singapore, Singapore

${ }^{3}$ Department of Psychosis, Institute of Mental Health, Singapore

${ }^{4}$ Office of Education, Duke-NUS Medical School, Singapore

${ }^{5}$ Department of Mood and Anxiety, Institute of Mental Health, Singapore

Correspondence: Dr Ying Ying Lee, Research Division, Institute of Mental Health, 10 Buangkok View, Buangkok Green Medical Park, Singapore 539747.

Email: ying_ying_lee@imh.com.sg
} 


\section{CLINICAL IMPACT}

\section{What is New}

- Self-reported dietary habits of people with depressive and psychotic disorders in Singapore were characterised.

\section{Clinical Implications}

- A host of sociodemographic factors, not diagnosis of mental disorders, influences the dietary quality of people with depressive and psychotic disorders.

- Clinicians treating psychiatric patients need to be aware of the nuanced reasons behind poor dietary choices and make targeted psychoeducation to specific subgroups within the patient population.

healthy dietary patterns reduce the risk of depression and that unhealthy dietary patterns elevate the risk of depression. ${ }^{10-12}$ However, it is unclear if a poor diet quality is prevalent among persons with known depressive disorders. ${ }^{4,13}$ Studies examining the association between diet quality and depression were commonly performed in the general population in whom the associations between diet and risk of depression or subclinical depressive symptoms, or both, were investigated; very few studies actually investigated the relationship between diet quality and clinically diagnosed depressive disorders. ${ }^{13-15}$

On the other hand, owing to the prevalence of cardiovascular risks, metabolic syndrome and obesity among persons with schizophrenia, much more attention has been paid to the diet and lifestyle habits of this population of patients. ${ }^{16-18}$ A number of studies linking weight and metabolic issues in persons with depression and schizophrenia were published in the last decade. ${ }^{19-21}$ For example, it was reported that people with schizophrenia spectrum disorder have poor diet quality, high refined carbohydrate intake and low intake of fibre. ${ }^{16,17,22,23}$ This finding could be in part due to the use of atypical antipsychotic medications, which affect neural pathways involved in appetite control and satiety. ${ }^{22}$ In addition, persons with schizophrenia spectrum disorder were more likely than the general population to have poorer dietary habits. ${ }^{16,17}$ Moreover, studies from Singapore and beyond have reported that a high proportion of persons with schizophrenia are overweight, obese and living with metabolic syndrome. ${ }^{17-20,22,24}$ Healthy diet has been identified as a potential preventive and/or adjunctive treatment for depressive and psychotic disorders. ${ }^{13,14,23}$

Given the concerning state of the physical health condition of persons with mental disorders, the Lancet Psychiatry Commission recently published a blueprint for protecting their physical health in an effort to improve the quality of life and prolong the lifespan of this vulnerable population. ${ }^{25}$ Five areas were identified, with modifiable factors in health-related behaviours (e.g. diet, smoking and physical activities) as key to protecting the physical health of persons with mental disorders. Yet, the extent to which lifestyle risk factors - such as diet-in various mental disorders differs from that in the general population is unknown. ${ }^{25}$

Most overseas studies that established the link between depressive symptoms and poor dietary patterns were conducted within the general population., ${ }^{4,13}$ In the same vein, most local studies that investigated the dietary patterns were conducted within the general population. ${ }^{26-28}$ To our knowledge, there is a paucity of data on the dietary patterns of patients with known mental disorders, especially those with depressive disorders. While there have been a lot of data internationally on the dietary patterns of persons with schizophrenia, there is a gap in the data in Singapore.

Given the ethnic diversity of Singapore, a culturally relevant diet screener needs to be developed and validated for use in the local population. Whitton et al. ${ }^{29}$ have developed and validated a short diet screener, which has been shown to have good reliability for use among Singapore residents. In another study, the same investigators found that Dietary Approaches to Stop Hypertension (DASH) was the most sensitive diet score to test associations between diet and health-related outcomes. ${ }^{30}$ Moreover, there exists prior literature that has linked adherence to DASH diet patterns to more positive mental health in various populations. ${ }^{31-33}$ While DASH was originally designed to prevent hypertension, it was suggested that hypertension and some mental disorders share common modifiable risk factors and underlying biological mechanisms. Also, DASH diet approach emphasises intake of nutrients with benefits to mental health, and lower intake of those detrimental to mental health. ${ }^{31}$

Our study aimed to (1) characterise the diet of a population of persons with depressive and psychotic disorders in Singapore and score their diet intake according to the DASH score; and (2) identify sociodemographic correlates of DASH scores among persons with depressive and psychotic disorders. 


\section{METHODS}

\section{Participants}

Singapore's population of 5.7 million people is made up of the 3 main ethnic groups of Chinese, Malay and Indian. During the period of October 2018 to February 2020, participants were recruited from the Institute of Mental Health, the only tertiary psychiatric hospital in Singapore. Participants comprising both inpatients and outpatients were included in the study if they were aged 21 to 65 years and had a clinician-reported diagnosis of either depressive disorders (e.g. major depressive disorder, dysthymia, depression with psychotic features) or psychotic disorders (e.g. brief psychotic disorder, schizophrenia and schizoaffective disorder). The study excluded participants who did not have the mental capacity to consent to the study, or did not meet the diagnostic criteria. Depending on the preference of the participants, the survey was administered in 1 of the 4 local languages: English, Chinese, Malay and Tamil. Data of this study came from a larger study on the prevalence and correlates of cigarette smoking among persons with mental disorders. ${ }^{34}$ Ethics approval was obtained from the Institutional Research Review Board and National Healthcare Group Domain Specific Review Board (Ref: 2018/00772). Written consent was taken from all the participants. A quota was set to recruit similar proportions of persons based on depressive and psychotic disorders, sex and age.

\section{Measures}

\section{Sociodemographic information, smoking habits, body mass index and diagnosis}

A modified version of Global Adult Tobacco Survey ${ }^{35}$ was used to capture data that included sociodemographic information (e.g. age, sex, ethnicity, education, housing and income) and questions regarding smoking status. The classification of smoker, non-smoker and past smoker was based on the definitions from the National Health Interview Survey. ${ }^{36}$ Participants who had smoked at least 100 cigarettes in their lifetime and were smoking at the time of the survey were classified as a smoker. Participants who had smoked at least 100 cigarettes but had quit smoking at the time of the survey were classified as former smokers. Those who had never smoked or had smoked less than 100 cigarettes in their lifetime were classified as non-smokers. ${ }^{37}$ For the purpose of data analysis and interpretation, ex-smokers were subsumed under the category for current smokers to form a new classification "ever-smokers" because of the small sample size and the need to keep to the convention set by the first article published for this project. ${ }^{34}$ At the end of every interview, height and weight were measured at the clinic of recruitment. Data were analysed using the body mass index (BMI) scores. BMI was further categorised into 4 groups based on World Health Organization guidelines for Asian populations: underweight $\left(<18.5 \mathrm{~kg} / \mathrm{m}^{2}\right)$, normal range $\left(\geq 18.5 \mathrm{~kg} / \mathrm{m}^{2}\right.$ and $\left.<23 \mathrm{~kg} / \mathrm{m}^{2}\right)$, overweight $\left(\geq 23 \mathrm{~kg} / \mathrm{m}^{2}\right.$ and $<27.5 \mathrm{~kg} /$ $\left.\mathrm{m}^{2}\right)$ and obese $\left(\geq 27.5 \mathrm{~kg} / \mathrm{m}^{2}\right) .{ }^{38}$ The clinical diagnosis of each participant was captured as indicated in the electronic medical records, which followed the criteria of the Diagnostic and Statistical Manual of Mental Disorders, 4th edition. ${ }^{39}$

\section{Diet screener}

The diet screener utilised in the present study was developed and validated by Whitton et al. ${ }^{29}$ with a Singapore population. It comprised a list of 30 food/beverage items, with a 10-point scale-ranging from "never/rarely" to " 6 or more times per day" - the frequency at which respondents consumed a particular item within the last 1 year. The diet screener was interviewer-administered. Standard serving sizes were indicated for each food/beverage item to facilitate this process. Intake frequencies were standardised to a number of servings per day for each food/beverage item. DASH scores were calculated to account for the intakes of fruit, vegetables, nuts and legumes, whole grains, red and processed meat, low fat dairy, and sweetened beverages. ${ }^{40}$ For each of these 7 DASH components, participants received a score from 1 to 5 corresponding to the quintile of intake they fall in, with reverse scoring for meat and sweetened beverages, and these 7 quintile scores were summed to form the overall DASH score.

\section{Statistical analyses}

All analyses were conducted using Stata software version 15.0 (StataCorp, College Station, US). Means and standard deviations are provided for continuous variables, while frequencies and percentages are presented for categorical variables. To examine the variables associated with DASH scores, a linear regression was conducted with the full sample and the following variables: age, sex, ethnicity, education, employment, personal income, BMI, smoking status and diagnosis. A linear regression was conducted separately for the depressive and psychotic disorders groups to further examine the associations between sociodemographic characteristics or smoking status and DASH. Respondents who had any missing values on any variables were removed listwise in the regression 
analyses: 3 participants had missing values on the height and weight variables, and 2 participants had missing values on the DASH questionnaire. Estimates of the associations between DASH scores and variables with more than 1 comparison (i.e. ethnicity, education, employment, income and BMI) in each regression model were adjusted with Bonferroni correction for multiple comparisons. Lastly, to further examine sex and age group differences in DASH components within the full sample and among the depressive and psychotic disorders groups, bivariate Mann-Whitney $\mathrm{U}$ tests were conducted. Statistical significance was set at the conventional $\alpha$ level of $P<0.05$ using 2-tailed tests.

\section{RESULTS}

\section{Sociodemographic and clinical characteristics of sample}

Sociodemographic and clinical characteristics of the sample are shown in Table 1. The sample consisted of 380 respondents; $46.6 \%$ (177) were diagnosed with depressive disorders, while $53.4 \%$ (203) had psychotic disorders. The mean age of the sample was $39.8 \pm 12.0$ years (range 21-65 years), and approximately half of the sample $(188,49.5 \%)$ were aged 21 to 40 . Of the sample, $55.3 \%$ were men and $73.4 \%$ were of Chinese ethnicity.

\section{DASH scores among psychiatric patients in IMH}

The mean and standard deviations of each of the 7 DASH components (fruit, vegetables, nuts and legumes, whole grains, red and processed meat, low fat dairy, and sweetened beverages) and of the overall DASH scores of the sample for the depressive and psychotic disorder groups are presented in Table 2. Results of the regression analyses of the full sample as well as the depressive and psychotic disorder groups are given in Table 3.

Within the full sample, there were no significant differences in DASH scores between the depressive and psychotic disorder groups $(\mathrm{I}=0.89)$. However, compared with those aged 21 to 40, those in the age range of 41 to 65 had significantly a higher DASH scores $(\mathrm{B}=1.94,95 \%$ confidence interval $[\mathrm{CI}]$ 0.91-2.96, $P<0.001)$. Women appeared to have higher DASH scores than men $(\mathrm{B}=1.09,95 \% \mathrm{CI}$ 0.07-2.11, $P=0.04)$. Those who were economically inactive (students, homemakers and retirees) had higher DASH scores than those unemployed $(\mathrm{B}=1.98,95 \%$ CI $0.006-3.96$, $P=0.049$ ) (Table 3).

Within the depressive disorder group, age remained significantly and positively associated with DASH scores, with those in the age range of 41 to 65 having higher scores $(\mathrm{B}=2.65,95 \%$ CI $1.08-4.22, P=0.001)$. Those who were economically inactive had better DASH scores than those who were unemployed $(\mathrm{B}=2.59$, 95\% CI 0.04-5.13, $P=0.045$ ).

In the psychotic disorder group, age was similarly associated with DASH scores-those aged 41 to 65 years were associated with higher scores $(\mathrm{B}=1.53,95 \%$ CI $0.06-3.00, P=0.04)$. Those who ever smoked were also associated with lower scores compared with those who had never smoked ( $\mathrm{B}=-1.93,95 \% \mathrm{CI}-3.70$ to -0.17 , $P=0.03$ ) (Table 3). Finally, we did not note significant associations between the BMI of the sample population with their dietary patterns (Table 3).

\section{Sex and age group differences in DASH component scores}

Descriptive statistics and Mann-Whitney $\mathrm{U}$ tests were performed to examine sex and age group differences in DASH component scores among the total sample, and depressive and psychotic disorder groups. Based on Mann-Whitney U tests, it appeared that within the full sample, women had higher intake of fruits $(P=0.02)$, whole grains $(P=0.01)$ and higher overall DASH scores $(P=0.001)$, but had lower intake of red and processed meat $(P=0.009)$ and sweetened beverages $(P=0.38)$ than men. Within the entire sample, adults aged 41 to 65 had lower intake of red and processed meat $(P=0.001)$ and sweetened beverages $(P=0.009)$ than those aged 21 to 40 .

Within those with depressive disorders only, women had higher intake of fruits $(P=0.02)$, lower intake of red and processed meat $(P=0.03)$ and higher overall DASH scores $(P=0.02)$ than men. Among those with psychotic disorders, women had higher intake of whole grains $(P=0.03)$, lower intake of sweetened beverages $(P=0.03)$ and higher DASH scores $(P=0.01)$ than men.

\section{DISCUSSION}

This study is among the first to examine the nutrition intake and DASH scores of a sample of psychiatric patients at the Institute of Mental Health in Singapore. Seven components of the DASH scores and the final DASH scores are presented in Table 2. Persons with depressive disorders had a mean DASH score of $21.3( \pm 4.2)$, while persons with psychotic disorders had a mean DASH score of $21.2( \pm 4.9)$. In addition, we observed that the ever-smokers group was more likely than the non-smokers group to report poor diet scores within our sample population. On the other hand, we found that older age, women and economically inactive 


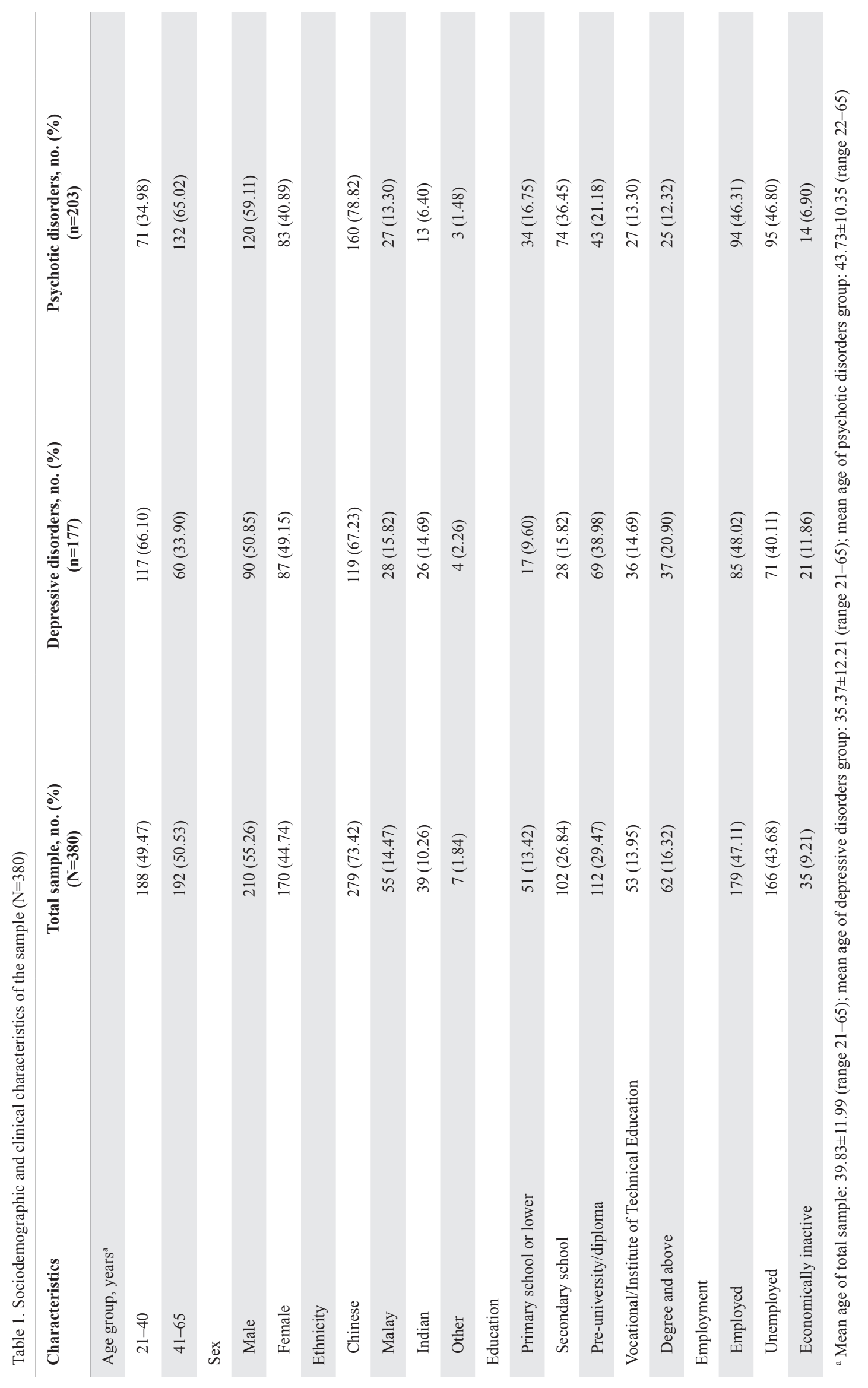




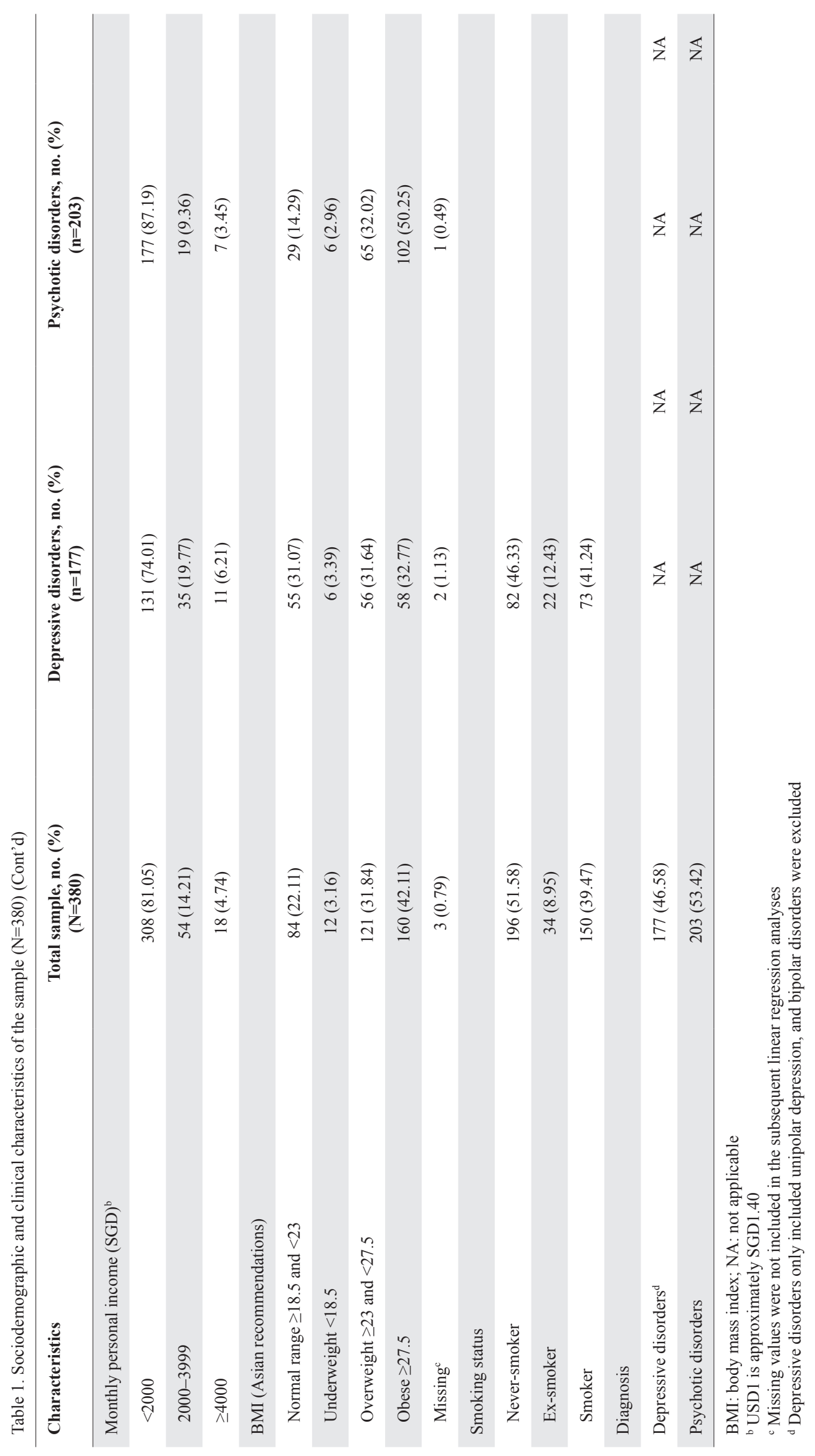




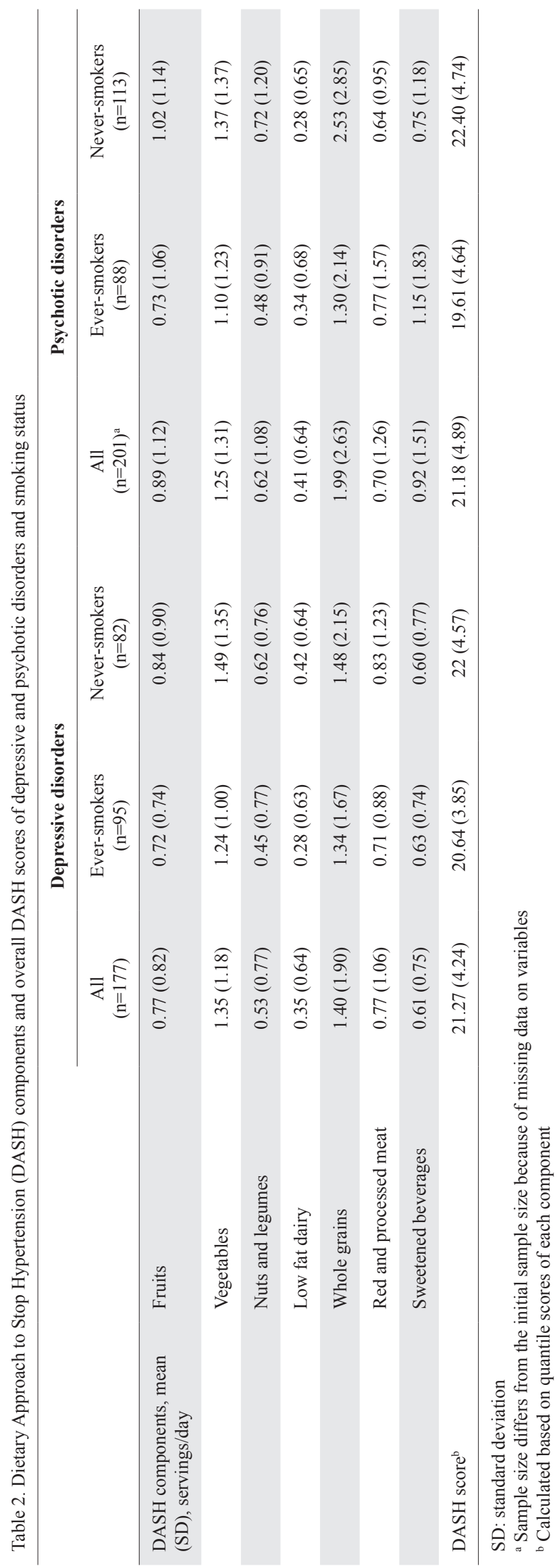

groups were more likely to report better diet quality than their respective reference groups (Table 3). We also observed age and sex differences in the intake of various food groups in our sample population.

When comparing the individual nutrition intake of our sample population with that of the general population from the National Nutrition Survey 2010, ${ }^{41}$ we found some interesting differences in the number of servings taken per day for various diet components. For instance, the wholegrain intake of our sample was much higher (1.34-2.53 servings per day) than the national average of 0.76 serving per day. ${ }^{41}$ A reason for this difference could be the success of Singapore's Health Promotion Board to encourage the intake of wholegrain staple food in its health promotion campaigns. On the other hand, the fruit and vegetable intake of our sample population was much lower than the reported average daily intake of the general population in Singapore. The National Nutrition Survey reported an intake of 1.27 servings of fruits and 1.78 servings of vegetables per day for adult Singapore residents. ${ }^{41}$ However, respondents of this present study reported a daily intake of fruits at $0.72-1.02$ servings and vegetables at 1.10-1.49 servings. This finding is in line with the literature, in which fruit and vegetable intake was reported to be lower among persons with schizophrenia. ${ }^{2}$

Findings from our study supported the notion that older adults, women and persons who are economically inactive adhere to a healthier diet based on their DASH scores. Persons who smoked were more likely to report a poor diet quality than those who did not. These findings are similar to the literature. Koh et al. reported that older Chinese adults in Singapore who smoked had poor dietary habits. ${ }^{26}$ It is well documented that persons who smoke have poor dietary habits. ${ }^{16,40}$ Reasons for poor diet choices could be that smokers are more likely than non-smokers to have a craving for high-fat food and fast food fats, and that nicotine dependence could have an effect on smokers' cravings for other substances like sugar. ${ }^{41}$ A study on fast food intake of Singapore residents found that younger adults tended to take more fast food than their older counterparts. ${ }^{28}$ In another Singapore study involving pregnant women, older women were found to have a healthier dietary pattern than younger women. It was suggested that older women may be more knowledgeable in nutrition and hence are able to make healthier dietary choices. ${ }^{27}$ In our study, we observed a similar trend that older people in the sample had higher DASH scores.

Diet is multifaceted, with the potential to be a preventive health measure or a modifiable health risk factor. From our study, we found a number of 


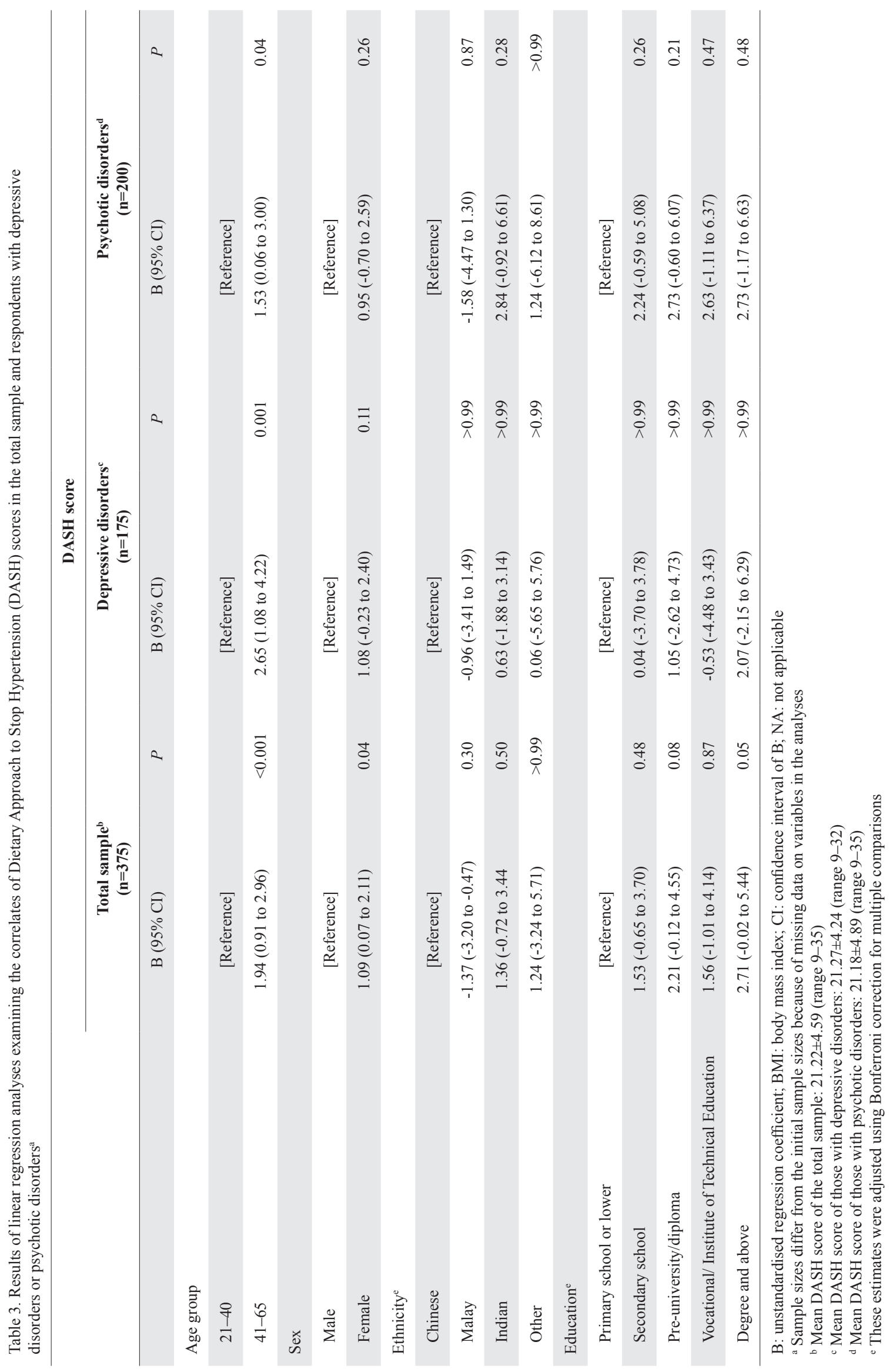




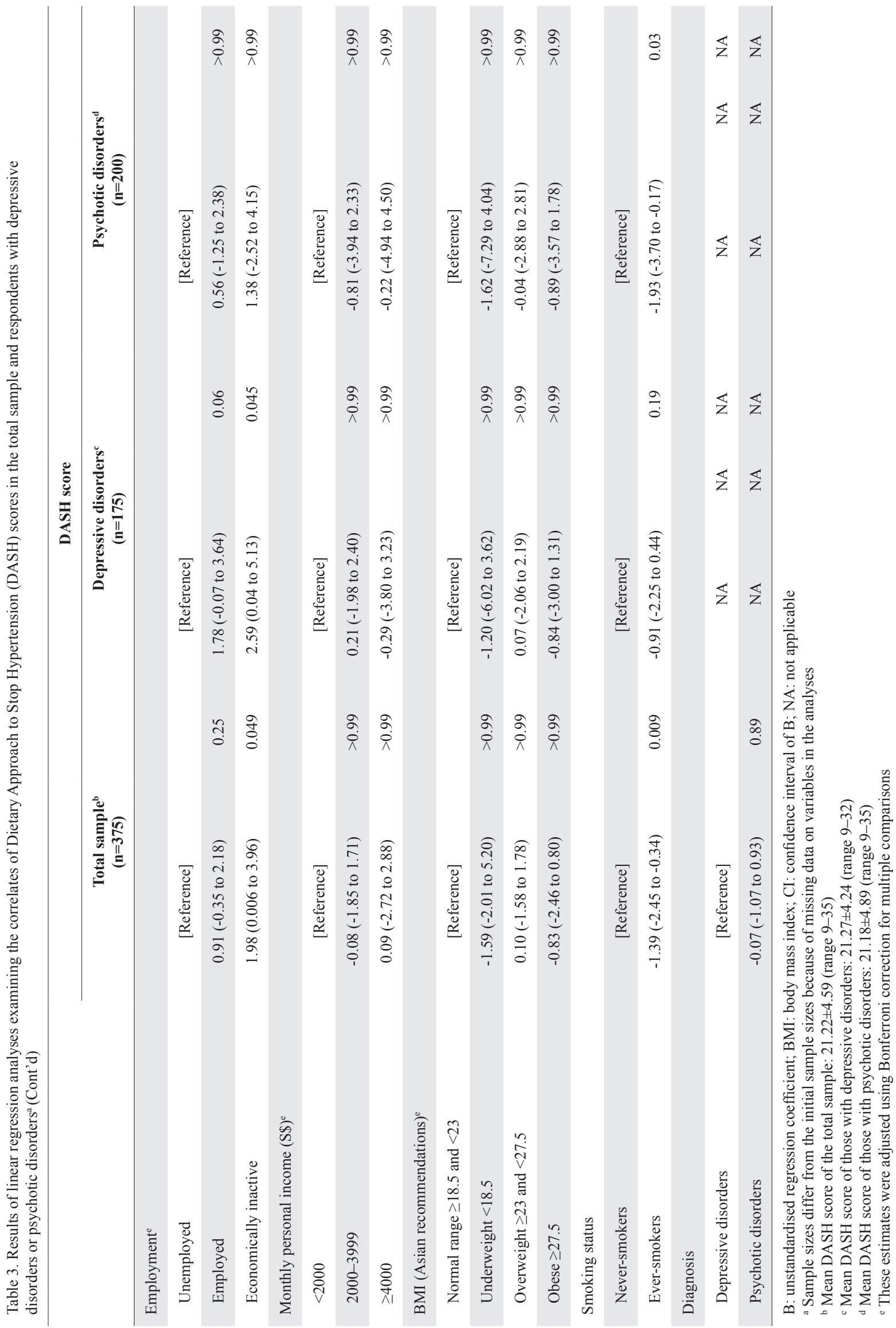


sociodemographic factors associated with dietary patterns in persons with depressive and psychotic disorders. Looking at the dietary choices of a sample population may not give an accurate reflection of the state of dietary choices of the actual population. Indeed, when we focused on specific groups within our sample population, we observed that smokers were more likely than non-smokers to report poorer diet quality. Older, female and economically inactive respondents were more likely to report having better diet quality than their counterparts. Our study highlights the role lifestyle choices plays in influencing the dietary choices of our population. Thus, the vulnerability to poor diet choices may not be attributed to the diagnosis of a mental disorder alone.

In the same vein, BMI was not found to be a factor associated with dietary habits. This is surprising because it has been noted in the literature that persons with schizophrenia have higher-than-normal $\mathrm{BMI}^{16}$ and people with depression have either lower- or higherthan-normal BMI. ${ }^{42,43}$ In line with the literature, there also seems to be a greater proportion of people classified as obese in the psychotic disorder group than in the depressive disorder group. It is well documented that atypical antipsychotic medications can cause metabolic syndrome in persons with psychotic disorders and that use of medications is associated with weight gain. ${ }^{20,24}$ Multiple reasons have been proposed for such weight gain, including the effect of antipsychotic drugs in neural pathways that regulate appetite control and energy metabolism. ${ }^{44}$ Since BMI did not predict diet quality, it seems that people with obesity in this sample were eating more in quantity but not necessarily poorer in quality than those who were non-obese. The weight disparity within the psychiatric population compared with the general population may be due to the treatment regimen prescribed to patients. On this note, treating physicians need to be fully aware of the unwanted physical health side effects that atypical antipsychotic medications can cause. ${ }^{20}$

There are several limitations in this study that could confound the findings. Firstly, the study sample was recruited by convenience sampling at a tertiary psychiatric hospital and may not be representative of the entire psychiatric population in Singapore. However, a quota was set to have a fair proportion of persons recruited into the study based on depressive and psychotic disorders, sex and age. Secondly, the diet screener was based on a self-reported diet recall of intake over the past year, with no validation using blood or urinary samples. There may be recall bias in the selfreport that we were unable to verify or refute by other means. Thirdly, while we gave the average serving sizes in our questionnaire in written form, we did not use visual representations to illustrate them as recommended. ${ }^{45}$ Fourthly, we did not make adjustments for total energy intake recommended for epidemiological-type studies. ${ }^{46}$ Finally, owing to the cross-sectional design of this study, we were unable to uncover a causal relationship between unhealthy diet and mental illness.

Given the study limitations, future work could focus on using a more representative sampling method to investigate the dietary habits of the psychiatric population in Singapore. Moreover, supplementing the diet recall questionnaire with photographs, or blood and/or urine sample analysis may also clarify any recall bias and errors. ${ }^{45}$ Furthermore, a host of sociodemographic factors such as lifestyle, sex and employment status, influences the dietary patterns of a given person. The significant comorbid condition of persons with mental disorders calls for innovative strategies to be implemented to alleviate the adverse outcomes and burden associated with the comorbidity. ${ }^{47}$

\section{Acknowledgement}

The authors are grateful to the participants of this study.

\section{REFERENCES}

1. World Health Organization. Diet, Nutrition and the Prevention of Chronic Diseases. Geneva: World Health Organization; 2003. WHO Technical Report Series No. 916.

2. Aucoin M, LaChance L, Cooley K, et al. Diet and psychosis: A scoping review. Neuropsychobiology 2020;79:20-42.

3. Jacka FN. Nutritional psychiatry: Where to next? EBioMedicine 2017;17:24-9.

4. Berk M, Jacka FN. Diet and depression-From confirmation to implementation. JAMA 2019;321:842-3.

5. Parletta N, Milte CM, Meyer BJ. Nutritional modulation of cognitive function and mental health. J Nutr Biochem 2013; 24:725-43.

6. Rao TS, Asha MR, Ramesh BN, et al. Understanding nutrition, depression and mental illnesses. Indian J Psychiatry 2008;50:77-82.

7. Park JH, Kim KW, Kim MH, et al. A nationwide survey on the prevalence and risk factors of late life depression in South Korea. J Affect Disord 2012;138:34-40.

8. Jacka FN, Pasco JA, Mykletun A, et al. Association of Western and traditional diets with depression and anxiety in women. Am J Psychiatry 2010;167:305-11.

9. Liu C, Xie B, Chou CP, et al. Perceived stress, depression and food consumption frequency in the college students of China Seven Cities. Physiol Behav 2007;92:748-54.

10. Lai JS, Hiles S, Bisquera A, et al. A systematic review and meta-analysis of dietary patterns and depression in communitydwelling adults. Am J Clin Nutr 2014;99:181-97.

11. Li Y, Lv MR, Wei YJ, et al. Dietary patterns and depression risk: A meta-analysis. Psychiatry Res 2017;253:373-82. 
12. Wang J, Zhou Y, Chen K, et al. Dietary inflammatory index and depression: A meta-analysis. Public Health Nutr 2019; 22:654-60.

13. Firth J, Marx W, Dash S, et al. The effects of dietary improvement on symptoms of depression and anxiety: A meta-analysis of randomized controlled trials. Psychosom Med 2019;81:265-80.

14. Quirk SE, Williams LJ, O'Neil A, et al. The association between diet quality, dietary patterns and depression in adults: A systematic review. BMC Psychiatry 2013;13:175.

15. Jacka FN, O’Neil A, Opie R, et al. A randomised controlled trial of dietary improvement for adults with major depression (the 'SMILES' trial). BMC Med 2017; 15:23.

16. McCreadie RG; Scottish Schizophrenia Lifestyle Group. Diet, smoking and cardiovascular risk in people with schizophrenia. Br J Psychiatry 2003;183:534-9.

17. Simonelli-Muñoz AJ, Fortea MI, Salorio P, et al. Dietary habits of patients with schizophrenia: A self-reported questionnaire survey. Int J Ment Health Nurs 2012;21:220-8.

18. Tsuruga K, Sugawara N, Sato Y, et al. Dietary patterns and schizophrenia: A comparison with healthy controls. Neuropsychiatr Dis Treat 2015;11:1115-20.

19. Subramaniam M, Picco L, He V, et al. Body mass index and risk of mental disorders in the general population: Results from the Singapore Mental Health Study. J Psychosom Res 2013;74:135-41.

20. Lee J, Nurjono M, Wong A, et al. Prevalence of metabolic syndrome among patients with schizophrenia in Singapore. Ann Acad Med Singap 2012;41:457-62.

21. de Wit LM, van Straten A, van Herten M, et al. Depression and body mass index, a U-shaped association. BMC Public Health 2009;9:14

22. Teasdale SB, Ward PB, Samaras K, et al. Dietary intake of people with severe mental illness: Systematic review and meta-analysis. Br J Psychiatry 2019;214:251-9.

23. Dipasquale S, Pariante CM, Dazzan P, et al. The dietary pattern of patients with schizophrenia: A systematic review. J Psychiatr Res 2013;47:197-207.

24. Lee J, Jiang J, Sim K, et al. Gender differences in Singaporean Chinese patients with schizophrenia. Asian J Psychiatr. 2011;4:60-4.

25. Firth J, Siddiqi N, Koyanagi A, et al. The Lancet Psychiatry Commission: A blueprint for protecting physical health in people with mental illness. Lancet Psychiatry 2019;6:675-712.

26. Koh WP, Yuan JM, Sun CL, et al. Middle-aged and older Chinese men and women in Singapore who smoke have less healthy diets and lifestyles than nonsmokers. J Nutr 2005;135:2473-7.

27. Han CY, Colega M, Quah EPL, et al. A healthy eating index to measure diet quality in pregnant women in Singapore: A cross-sectional study. BMC Nutr 2015;1:39.

28. Whitton C, Ma Y, Bastian AC, et al. Fast-food consumers in Singapore: Demographic profile, diet quality and weight status. Public Health Nutr 2014;17:1805-13.

29. Whitton C, Ho JCY, Rebello SA, et al. Relative validity and reproducibility of dietary quality scores from a short diet screener in a multi-ethnic Asian population. Public Health Nutr 2018; 21:2735-43.

30. Whitton C, Rebello SA, Lee J, et al. A healthy Asian a posteriori dietary pattern correlates with a priori dietary patterns and is associated with cardiovascular disease risk factors in a multiethnic Asian population. J Nutr 2018;148:616-23.
31. Valipour G, Esmaillzadeh A, Azadbakht L, et al. Adherence to the DASH diet in relation to psychological profile of Iranian adults. Eur J Nutr 2017;56:309-20.

32. Perez-Cornago A, Sanchez-Villegas A, Bes-Rastrollo M, et al. Relationship between adherence to Dietary Approaches to Stop Hypertension (DASH) diet indices and incidence of depression during up to 8 years of follow-up. Public Health Nutr 2017; 20:2383-92.

33. Faghih S, Babajafari S, Mirzaei A, et al. Adherence to the Dietary Approaches to Stop Hypertension (DASH) dietary pattern and mental health in Iranian university students. Eur J Nutr 2020;59:1001-11

34. Asharani PV, Seet VAL, Abdin E, et al. Smoking and mental illness: Prevalence, patterns and correlates of smoking and smoking cessation among psychiatric patients. Int J Environ Res Public Health 2020;17:5571

35. Asma S, Mackay J, Song SY, et al. The GATS Atlas: Global Adult Tobacco Survey. Atlanta, GA: CDC Foundation; 2015.

36. McClave AK, McKnight-Eily LR, Davis SP, et al. Smoking characteristics of adults with selected lifetime mental illnesses: Results from the 2007 National Health Interview Survey. Am J Public Health 2010;100:2464-72.

37. Centers for Disease Control and Prevention. National Health Interview Survey Glossary, 29 August 2017. Available at: https:// www.cdc.gov/nchs/nhis/tobacco/tobacco_glossary.htm. Accessed on 7 January 2020.

38. WHO Expert Consultation. Appropriate body-mass index for Asian populations and its implications for policy and intervention strategies. Lancet 2004;363:157-63.

39. American Psychiatric Association. Diagnostic and Statistical Manual of Mental Disorders, Text Revision (DSM-IV-TR), 4th edition. Washington, DC: American Psychiatric Association Publishing; 2000.

40. Fung TT, Chiuve SE, McCullough ML, et al. Adherence to a DASH-style diet and risk of coronary heart disease and stroke in women. Arch Intern Med 2008;168:713-20.

41. Health Promotion Board. National Nutrition Survey 2010. Singapore: Health Promotion Board; 2013. Available at: https:// www.hpb.gov.sg/docs/default-source/pdf/nns-2010-report. pdf?sfvrsn=18e3f172 2. Accessed on 5 October 2020 .

42. Lin $\mathrm{CH}$, Chen $\mathrm{CC}$, Wong $\mathrm{J}$, et al. Both body weight and BMI predicts improvement in symptom and functioning for patients with major depressive disorder. J Affect Disord 2014;161:123-6.

43. Opel N, Redlich R, Grotegerd D, et al. Obesity and major depression: Body-mass index (BMI) is associated with a severe course of disease and specific neurostructural alterations. Psychoneuroendocrinology 2015;51:219-26

44. Dayabandara M, Hanwella R, Ratnatunga S, et al. Antipsychoticassociated weight gain: Management strategies and impact on treatment adherence. Neuropsychiatr Dis Treat 2017;13:2231-41.

45. Naska A, Lagiou A, Lagiou P. Dietary assessment methods in epidemiological research: Current state of the art and future prospects. F1000Res 2017;6:926.

46. Willett WC, Howe GR, Kushi LH. Adjustment for total energy intake in epidemiologic studies. Am J Clin Nutr 1997;65(4 Suppl): 1220S-1228S

47. Dash SR, O'Neil A, Jacka FN. Diet and common mental disorders: The imperative to translate evidence into action. Front Public Health 2016;4:81. 